EPJ Web of Conferences 41, 08019 (2013)

DOI: $10.1051 /$ epjconf/20134108019

(C) Owned by the authors, published by EDP Sciences, 2013

\title{
Ultrafast Charge Transfer Visualized by Two-Dimensional Electronic Spectroscopy
}

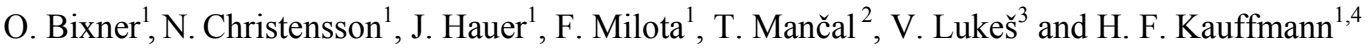 \\ ${ }^{1}$ Faculty of Physics, University of Vienna, Strudlhofgasse 4, 1090 Vienna, Austria \\ ${ }^{2}$ Institute of Physics, Charles University, Ke Karlovu 5, Prague 121 16, Czech Republic \\ ${ }^{3}$ Department of Chemical Physics, Slovak Technical University, Radlinského 9, \\ 81237 Bratislava, Slovakia \\ ${ }^{4}$ Faculty of Physics, Vienna University of Technology, Wiedner Hauptstrasse 8-10, \\ 1040 Vienna, Austria
}

\begin{abstract}
Two-dimensional electronic spectroscopy (2D-ES) is used to investigate ultrafast excited-state dynamics in a lutetium bisphthalocyanine dimer. Following optical excitation, a chain of electron and hole transfer steps gives rise to characteristic cross-peak dynamics in the electronic 2D spectra. The combination of density matrix propagation and quantum chemical calculations results in a molecular view of the charge transfer dynamics and highlights the role of the counter-ion in providing an energetic perturbation which promotes charge transfer across the complex.
\end{abstract}

\section{Introduction}

Charge transfer (CT) is integral to a diversity of chemical reactions and bioenergetic pathways [1]. Due to their low transition dipole moments, CT states can only participate in photo-induced reactions via the interaction with optically allowed locally excited (LE) states. The mixing of LE and CT states is determined by the interplay of resonance- (Columbic interaction) and CT-coupling (depending on wave function overlap) [2]. In this work we study the combined influence of CT- and resonancecoupling in a metal bridged bisphthalocyanine dimer ([LuPc$\left.\left.c_{2}\right]\right)$, and investigate how it modulates the electronic structure and excited state dynamics. In addition, we show how association with a counterion provides a driving force for $\mathrm{CT}$ across the complex following optical excitation.

\section{Results}

Figure 1 shows the linear absorption spectrum of $\left[\mathrm{LuPc}_{2}\right]$ in its neutral (radical) $\left(\left[\mathrm{LuPc}_{2}\right]^{\circ}\right)$ and anionic forms $\left(\left[\mathrm{LuPc}_{2}\right]^{-}\right)$. In the neutral form, the single transition in the linear absorption spectrum can be understood as a transition to the upper state in an H-type dimer formed by resonance coupling between the LEs on each of the rings. For the anion, configuration interaction between inter-ring CTand LE-states yields a double peak structure in the linear absorption spectrum [3]. For a symmetric complex, each transition is two- (anion) or four-fold (radical) degenerate.

This is an Open Access article distributed under the terms of the Creative Commons Attribution License 2.0, which permits unrestricted use, distribution, and reproduction in any medium, provided the original work is properly cited. 


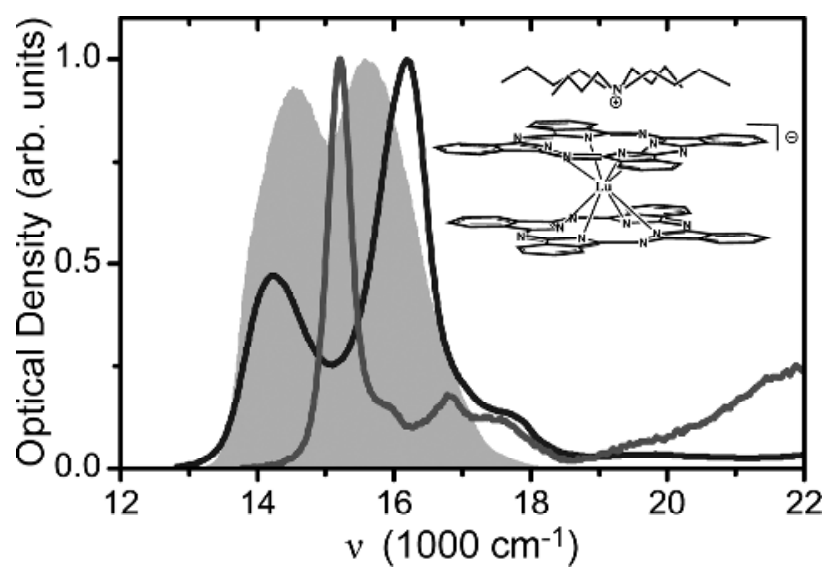

Fig. 1. Linear absorption spectra of $\left[\mathrm{LuPc}_{2}\right]^{-}$(solid black) and $\left[\mathrm{LuPc}_{2}\right]^{\bullet}$ (grey) together with the spectrum of the pulses used in the experiment (grey shaded area). The inset shows the structure of the $\left[\mathrm{LuPc}_{2}\right]^{-}$complex and the position of the $\mathrm{TBA}^{+}$counter-ion.

The two-dimensional spectra of $\left[\mathrm{LuPc}_{2}\right]^{-} \mathrm{TBA}^{+}$, the tetrabutylammonium salt of the anionic complex in benzonitrile, are shown in Figure 2 for selected waiting times $\left(\mathrm{t}_{2}\right)$. The $2 \mathrm{D}$ spectra reveal two diagonal peaks corresponding to the two main bands in the linear absorption spectrum. In addition, there is one cross-peak above the diagonal. However, contrary to what would be expected for a coupled dimer, there is no corresponding cross-peak below the diagonal. For longer $t_{2}$, the cross-peak below the diagonal recovers. The lack of a cross-peak at short times is readily reproduced in the simulation of the 2D spectra (Figure 2), and we can assign this to cancellation between excited state absorption from the upper band (negative) and the ground state bleach (positive) [4].

A closer inspection of the $2 \mathrm{D}$ spectrum for $t_{2}=45$ fs shows that the low energy band comprises two transitions. In addition, the two cross-peaks below the diagonal (cp1 and cp2) show distinct dynamics. The amplitude of cp2 rises on a $30 \mathrm{fs}$ timescale, while the amplitude of cp1 shows a delayed rise. Neither the double peak structure of the low energy band nor the distinct cross-peak dynamics are predicted by the exciton model previously used to describe [ $\left.\mathrm{LuPc}_{2}\right]^{-}[3]$. To find the origin of these additional transitions, we investigated the influence of the counter-ion on the electronic structure by TD-DFT calculations [4]. We found that the association with the counter-ion serves to stabilize CT states representing charge migration towards the counter-ion and that steric interactions break the energetic degeneracy of each transition found in the exciton model. The calculations show that the two transitions in the low energy band terminate in LUMO orbitals where the excited state charge density is located on different sides of the complex (Figure 2). After photoexcitation, relaxation from the high energy band leads to the formation of the high energy cross-peak denoted cp2 in Figure 2. Further relaxation, on a $30 \mathrm{fs}$ timescale, leads to the formation of cp1. Based on the orbitals in Figure 2, we can conclude that this relaxation step (cp2 to cp1) reflects the electron transfer step away from the counter-ion. Subsequent relaxation (on a $100 \mathrm{fs}$ ) timescale leads to the formation of a stable (on a ps timescale) charge separated excited state where the excited state charge density is located on the ring closet to the counter-ion.

The mixing of CT- and LE-states has a drastic influence on the electronic structure of the complex (Figure 1). The same holds true for the excited state dynamics. The anion displays a complicated pattern of electron and hole transfer steps which proceed on $30 \mathrm{fs}$ timescale. The radical on the other hand exhibits significantly slower excited state dynamics. Upon excitation, the bright state decays into the lowest exciton state with a $400 \mathrm{fs}$ time constant. The order of magnitude difference in relaxation dynamics is not related to the stronger system-bath coupling of the CT states, but due to the smaller separation between states in the electronic structure of the anion. The smaller energetic difference between the electronic states in the anion enables efficient relaxation via the high frequency intra-molecular vibrational modes of the monomers [4]. 

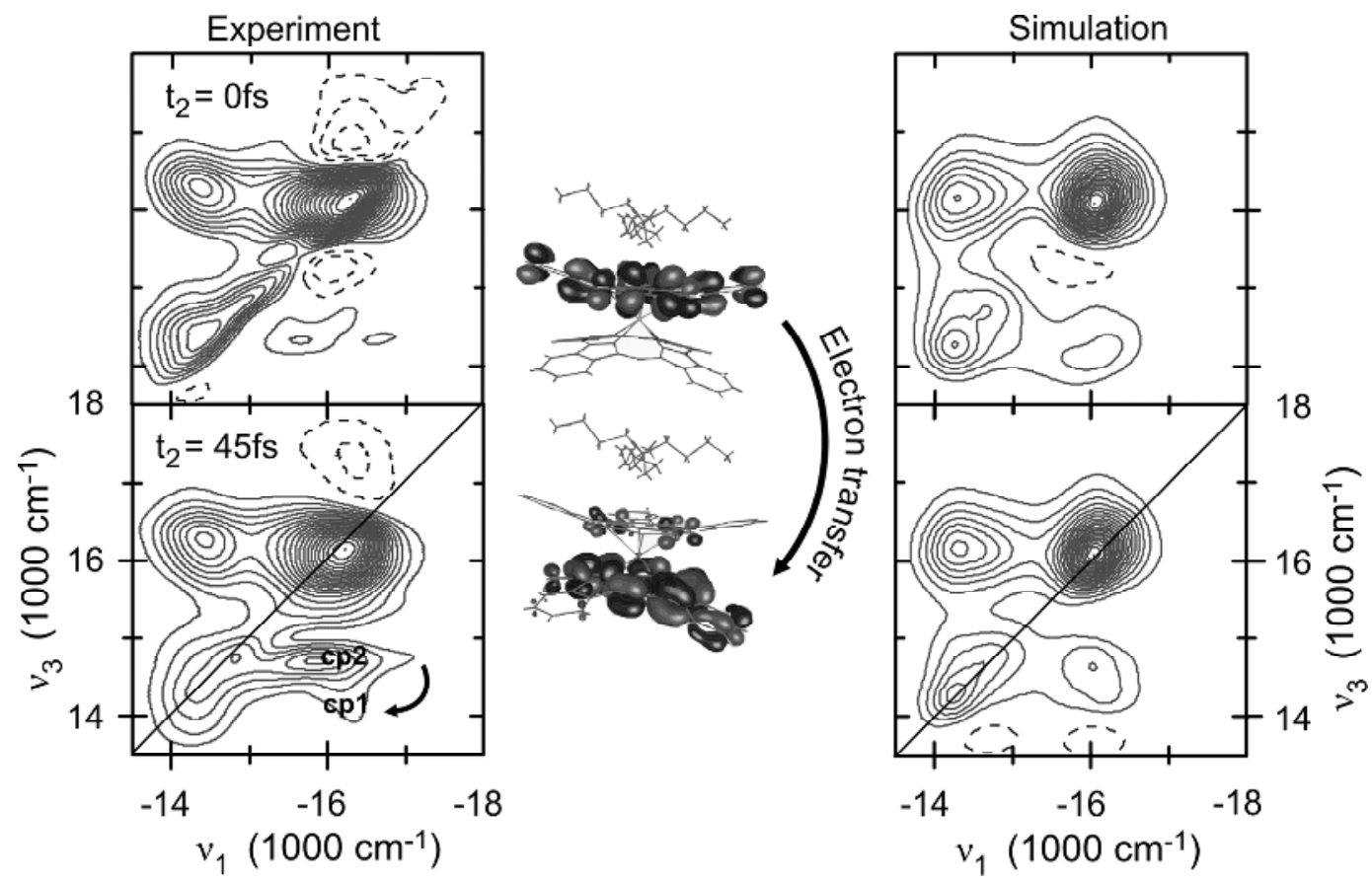

Fig. 2. Two-dimensional spectra of $\left[\mathrm{LuPc}_{2}\right]^{-}$at waiting times indicated in the figure. The simulations are shown on the right. The orbitals in the middle reflect the most prominent contributions to the transition in the low energy band. The relaxation from $\mathrm{cp} 1$ to $\mathrm{cp} 2$ reports on electron transfer from the macrocycle closest to the counter-ion to the other side of the complex.

\section{Conclusions}

In this work we have investigated the excited state dynamics in a lutetium bisphthalocyanine dimer exhibiting significant resonance- and CT-couplings. The resulting mixing of CT- and LE-states, as well as the association with a counter-ion, is found to have a profound impact on the electronic structure and excited state dynamics in the complex. Following optical excitation, CT between the phthalocyanine rings is found to proceed on a $30 \mathrm{fs}$ timecale. The inter-ring $\mathrm{CT}$ is enabled by the asymmetric interaction with the counter-ion, which stabilizes CT states representing CT towards the phthalocyanine ring closest to the counter-ion. The final charge separated state (excited state charge density closest to the counter-ion) is reached after a series of electron and hole transfer steps. Combining the evolution of the $2 \mathrm{D}$-spectra with density matrix propagation and quantum chemical calculations provides a detailed molecular and temporal view on the ultrafast $\mathrm{CT}$ dynamics in the complex.

\section{References}

[1] R. A. Marcus, N. Sutin Biochim. Biophys., 811, 265 (1985)

[2] V. May, O. Kühn Charge and Energy Transfer Dynamics in Molecular Systems, (Wiley-VCH, Berlin, 2000)

[3] N. Ishikawa, O. Ohno, Y. Kaizu, H. Kobayashi J. Phys. Chem., 96, 8832 (1992)

[4] O. Bixner, V. Lukes, T. Mancal, J. Hauer, F. Milota, M. Fischer, I. Pugliesi, M. Bradler, W.

Schmid, E. Riedle, H. F. Kauffmann, N. Christensson J. Chem. Phys., 136, 204503 (2012) 